\title{
PRESSURE WAVES IN SNOW
}

\author{
By R. L. BRown* \\ (U.S. Army Cold Regions Research and Engineering Laboratory, Hanover, New \\ Hampshire 03755 , U.S.A.)
}

\begin{abstract}
A dynamic constitutive law is used to study the response of medium-density snow to shock waves. The results show good correlation between theory and experiment, except for low-intensity shocks which produce small permanent density changes. In this case the validity of the data is questioned, although further experimental work is needed to settle this question. The results of this work also partially explain why snow is so effective in absorbing energy associated with stress waves. This is felt to be due to the workhardening characteristics of snow.

RÉsumé. Ondes de pression dans la neige. Une loi dynamique est utilisée pour étudier la réponse d'une neige de densité moyenne à des ondes de choc. Les résultats montrent une bonne corrélation entre la théorie et l'expérience sauf pour les chocs de faible intensité qui produisent de petits changements permanents de densité. Dans ce cas, la validité des résultats est mise en question bien que de nouvelles expériences soient nécessaires pour résoudre cette difficulté. Les résultats de ce travail expliquent aussi en partie pourquoi la neige est si efficace pour absorber l'énergie associée aux ondes de contrainte. On pense que ce résultat est dû aux caractéristiques d'un durcissement de la neige coûteux en énergie.

Zusammenfassung. Druckwellen im Schnee. Zum Studium der Reaktion von Schnee mittlerer Dichte auf Stosswellen wird ein dynamisches Grundgesetz herangezogen. Die Ergebnisse zeigen gute Übereinstimmung zwischen Theorie und Versuch, mit Ausnahme für Stösse geringer Intensität, die kleine, dauernde Dichteänderungen hervorbringen. In diesem Fall sind die Daten zweifelhaft; zur Klärung dieser Frage sind weitere Versuche notwendig. Die Ergebnisse der Arbeit erklären teilweise auch, warum Energie aus Druckwellen von Schnee so gut absorbiert werden kann. Es wird angenommen, dass dies eine Folge der Härtungscharakteristiken von Schnee ist.
\end{abstract}

\section{INTRODUGTION}

In an earlier paper Brown (in press) developed a dynamic volumetric constitutive law to represent the behavior of snow subjected to pressure loading. That law was shown to be capable of representing material behavior for a wide range of deformation rates. However, in the previous paper only quasi-static load conditions were discussed in detail and compared to experimental results of Abele and Gow (1975, 1976). Under these conditions the constitutive law could accurately represent the compaction of medium-to-high-density snow for strain-rates as low as $10^{-5} \mathrm{~s}^{-1}$.

The development of the constitutive law was based on the concept of pore collapse, an idea used earlier by Carroll and Holt (1972, 1973) for porous aluminum. Physically, the material is modeled as a suspension of voids or pores in a matrix material (in this case, polycrystalline ice) which was assumed to be incompressible, so that any changes in density result entirely from alteration of pore volume. The matrix material was represented as an elasticviscoplastic material with a rate-sensitive yield stress. Work-hardening effects were also included in this formulation. The resulting constitutive equation for snow is

$$
\hat{p}(t)=\frac{f}{3 \alpha} \ln \left(\frac{\alpha}{\alpha-\mathrm{I}}\right)\left\{2\left(S_{0}-C\right)+C \ln \left[\frac{(-A \dot{\alpha})^{2}}{\alpha(\alpha-\mathrm{I})}\right]\right\} \exp \left(-\phi \alpha / \alpha_{0}\right)-\frac{\gamma^{2}}{2 \alpha} \frac{\mathrm{d}}{\mathrm{d} \alpha}\left[\dot{\alpha}^{2} \mathrm{f}(\alpha)\right],
$$

where

$$
\begin{aligned}
\gamma^{2} & =\rho_{\mathrm{m}} a_{0}{ }^{2} /\left[3\left(\alpha_{0}-1\right)^{\frac{2}{2}}\right], \\
\mathrm{f}(\alpha) & =(\alpha-1)^{-\frac{1}{3}}-\alpha^{-\frac{1}{3}},
\end{aligned}
$$

* On sabbatical leave from Civil Engineering and Engineering Mechanics Department, Montana State University, Bozeman, Montana 597 I 7 , U.S.A. 
$\hat{p}$ is the pressure, and $S_{0}, C$, and $A$, are material constants which characterize the mechanical properties of ice. $\mathcal{F}$ and $\phi$ are work-hardening terms. For a detailed discussion of the development of the above equation, the reader is referred to Brown (in press). The density ratio $\alpha$ is just the ratio $\rho_{\mathrm{m}} / \rho$ of the mass density of ice $\rho_{\mathrm{m}}$ to the mass density of snow $\rho . a_{0}$ is the initial pore radius, and $\alpha_{0}$ is the initial density ratio of the material. As can be judged by the constitutive law, the pressure response is divisible into two parts, a quasi-static part given by the first term on the right-hand side of the equation and a dynamic part reflecting the pressure required to produce a given amount of kinetic energy.

At very large rates of compaction, Brown (in press) indicated that the yield stress of ice becomes weakly rate-dependent. As a consequence this allows some simplification of the constitutive law for snow. Under these assumptions, Equation (I) reduces to

$$
\hat{p}=\frac{2 \Upsilon_{0} \mathcal{J}}{3^{\alpha}} \ln \left(\frac{\alpha}{\alpha-\mathrm{I}}\right) \exp \left(-\phi \alpha / \alpha_{0}\right)-\frac{\gamma^{2}}{2 \alpha} \frac{\mathrm{d}}{\mathrm{d} \alpha}\left[\dot{\alpha}^{2} \mathrm{f}(\alpha)\right]
$$

where $Y_{0}$ is a material constant.

In this paper, we consider the application of Equations (I) and (4) to the study of stress waves. A more general solution utilizing Equation ( $\mathrm{I}$ ) will be developed first, as this solution is considered to be valid for a wide range of compressive stress waves. Then the more restrictive Equation (4) will be used to study shock waves, which generally involve very high strain-rates. The theory is then compared with the experimental data of Napadensky (1964) who conducted an extensive series of experiments on plastic waves in snow. Mellor ([1975], 1977) found that some of these data contained errors but did rework the data into a usable form.

\section{AnAlysis}

\section{Stress waves}

Generally when snow is impacted with enough intensity to generate a wave, two waves will propagate from the impacted zone. An elastic precursor wave precedes the plastic wave and travels at a speed that may be several times faster than that of the plastic wave. Since the elastic wave is generally quite weak, we neglect its effects and consider the plastic wave to be entering an undeformed medium.

The equation of motion for a wave propagating in the $X$-direction is

$$
\nabla_{X} \cdot \mathbf{T}^{0}+\rho_{0} b_{0}=\rho_{0} \frac{\mathrm{d}^{2} x}{\mathrm{~d} t^{2}},
$$

where $x$ is the deformed coordinate, $X$ is the undeformed coordinate vector, To is the first Piola stress tensor, $b_{0}$ is a body force, and $\rho_{0}$ is the initial material density. Assuming the plane wave is propagating in the $X$ direction, this equation reduces to

$$
-\frac{\partial \hat{p}}{\partial X}=\rho_{0} \frac{\partial v}{\partial t}
$$

where $\hat{p}$ is the hydrostatic pressure, and $v$ is the particle velocity in the $X$ direction. The continuity equation is

$$
\frac{\mathrm{d}}{\mathrm{d} t}(\rho f)=\mathrm{o},
$$

where $\rho$ is the instantaneous density and $\mathcal{f}$ is the Jacobian of the deformation. This reduces to

$$
\frac{\partial v}{\partial X}=\frac{1}{\alpha_{0}} \frac{\partial \alpha}{\partial t} \text {. }
$$


If the strèss wave is steady and propagating with a velocity $C_{0}$, the pressure, particle velocity, and density ratio can be defined in terms of the single variable

$$
\eta=X-C_{0} t .
$$

Substituting this into the equation of motion and the continuity equation gives, respectively,

$$
\begin{aligned}
& \frac{\mathrm{d} \hat{p}}{\mathrm{~d} \eta}=\rho_{0} C_{0} \frac{\mathrm{d} v}{\mathrm{~d} \eta}, \\
& \frac{\mathrm{d} v}{\mathrm{~d} \eta}=-\frac{C_{0}}{\alpha_{0}} \frac{\mathrm{d} \alpha}{\mathrm{d} \eta} .
\end{aligned}
$$

Integration of these equations gives the jump equations which define the increase of $\hat{p}$ and $v$ across the plastic wave, or

$$
\begin{aligned}
& \Delta \hat{p}=\rho_{0} C_{0} \Delta v, \\
& \Delta v=-\frac{C_{0}}{\alpha_{0}} \Delta \alpha .
\end{aligned}
$$

Combining these two, the pressure jump becomes

$$
\Delta \hat{p}=-\frac{\rho_{0} C_{0}^{2}}{\alpha_{0}} \Delta \alpha .
$$

If the material is at rest just prior to the arrival of the stress wave, then the jumps $\Delta \hat{p}$ and $\Delta v$ equal the values of $\hat{p}$ and $v$ as the wave passes. Denoting the steady values of $\alpha$ and $\hat{p}$ behind the wave by the star ${ }^{\star}$, we can write

$$
\begin{aligned}
\alpha^{\star} & =\alpha_{0}-\frac{\alpha_{0}}{\rho_{0} C_{0}{ }^{2}} \hat{p}^{\star}, \\
v^{\star} & =\frac{\mathrm{I}}{\rho_{0} C_{0}} \hat{p}^{\star} .
\end{aligned}
$$

From here on a star will denote the value of a variable behind the wave, i.e. in the steady condition after the wave has passed. The terms $\Delta v, \Delta \alpha$, and $\Delta \hat{p}$ refer to the values of the respective jumps at any point in the wave.

Now consider the constitutive equation once again. Substituting Equation (I) into Equation (14) and assuming that the material is initially unstressed gives

$$
\begin{aligned}
-\frac{\rho_{0} C_{0}{ }^{2}}{\alpha_{0}}\left(\alpha-\alpha_{0}\right)=\frac{f}{3 \alpha} \exp \left(-\phi \alpha / \alpha_{0}\right) & \ln \left(\frac{\alpha}{\alpha-\mathrm{I}}\right)\left\{2\left(S_{0}-C\right)+\right. \\
& \left.+C \ln \left[\frac{\left(A C_{0} \alpha^{\prime}\right)^{2}}{\alpha(\alpha-\mathrm{I})}\right]\right\}-\frac{C_{0}{ }^{2} \gamma^{2}}{2 \alpha} \frac{\mathrm{d}}{\mathrm{d} \alpha}\left[\left(\alpha^{\prime}\right)^{2} \mathrm{f}(\alpha)\right] .
\end{aligned}
$$

The prime denotes differentiation with respect to the variable $\eta$. Integrating the above results in

$$
\begin{aligned}
& -\frac{\rho_{0} C_{0}{ }^{2}}{3 \alpha_{0}}\left(\alpha-\alpha_{0}\right)\left[\alpha^{2}-\frac{\alpha_{0}}{2}\left(\alpha+\alpha_{0}\right)\right]=-\frac{C_{0}^{2} \gamma^{2}}{2} \Delta\left[\left(\alpha^{\prime}\right)^{2} \mathrm{f}(\alpha)\right]+ \\
& +\int_{\alpha_{0}}^{\alpha} \frac{7}{3} \ln \left(\frac{\alpha}{\alpha-1}\right)\left\{2\left(S_{0}-C\right)+C \ln \left[\frac{\left(A C_{0} \alpha^{\prime}\right)^{2}}{\alpha(\alpha-1)}\right]\right\} \exp \left(-\phi \alpha / \alpha_{0}\right) \mathrm{d} \alpha .
\end{aligned}
$$

The above equation describes the jump in density ratio across the wave, once the wave speed is known. However, this is an integro-differential equation and would have to be solved by a numerical method or some other approximate method.

In cases where strain-rates are not extremely large, this equation would have to be solved. Such would be the case for low-frequency, large-amplitude waves. 
If one is considering the case of shock waves, Equation (4) can be used in place of Equation (I), since shock waves generally produce exceptionally large strain-rates. Substitution of Equation (4) into Equation (14) results in

$$
-\frac{\rho_{0} C_{0}^{2}\left(\alpha-\alpha_{0}\right)}{\alpha_{0}}=\frac{{ }_{2} \Upsilon_{0}}{3 \alpha} \mathcal{J} \ln \left(\frac{\alpha}{\alpha-1}\right) \exp \left(-\phi \alpha / \alpha_{0}\right)-\frac{C_{0}{ }^{2} \gamma^{2}}{2 \alpha} \frac{\mathrm{d}}{\mathrm{d} \alpha}\left[\left(\alpha^{\prime}\right)^{2} \mathrm{f}(\alpha)\right],
$$

or, upon integrating,

$$
\begin{aligned}
-\frac{\rho_{0} C_{0}^{2}\left(\alpha-\alpha_{0}\right)}{3 \alpha_{0}}\left[\alpha^{2}-\frac{\alpha_{0}}{2}\left(\alpha+\alpha_{0}\right)\right]=- & \frac{C_{0}^{2} \gamma^{2}}{2} \Delta\left[\left(\alpha^{\prime}\right)^{2} \mathrm{f}(\alpha)\right]+ \\
& +\int_{\alpha_{0}}^{\alpha} \frac{27 Y_{0}}{3} \ln \left(\frac{\alpha}{\alpha-1}\right) \exp \left(-\phi \alpha / \alpha_{0}\right) \mathrm{d} \alpha .
\end{aligned}
$$

In steady waves, $\alpha^{\prime}=0$ both ahead of and behind the wave, so that, Equation (20) can be used to calculate the pressure jump across the wave: Substituting Equation (14) into Equation (20) for steady waves gives

$$
\hat{p}^{\star}=\left[2 \mathcal{J} Y_{0} \int_{\alpha_{0}}^{\alpha^{\star}} \ln \left(\frac{\alpha}{\alpha-1}\right) \exp \left(-\phi \alpha / \alpha_{0}\right) \mathrm{d} \alpha\right]\left[\alpha^{\star 2}-\frac{\alpha_{0}}{2}\left(\alpha^{\star}+\alpha_{0}\right)\right]^{-1},
$$

carrying out the indicated integration results in

$$
\hat{p}^{\star}=\left[\alpha^{\star 2}-\frac{\alpha_{0}}{2}\left(\alpha^{\star}+\alpha_{0}\right)\right]^{-1}\left\{g_{\mathrm{I}}\left(\alpha^{\star}\right)-\exp \left(-\phi \mid \alpha_{0}\right) g_{\mathrm{I}}\left(\alpha^{\star}-\mathrm{I}\right)\right\},
$$

where

$$
\begin{aligned}
g_{\mathrm{I}}\left(\alpha^{\star}\right)=-\frac{\alpha_{0}}{\phi}\left[\exp \left(-\phi \alpha^{\star} / \alpha_{0}\right) \ln \alpha^{\star}-\right. & \left.\exp (-\phi) \ln \alpha_{0}\right]+ \\
& +\frac{\alpha_{0}}{\phi}\left[\ln \frac{\alpha^{\star}}{\alpha_{0}}+\sum_{i=1}^{\infty} \frac{(-\phi)^{n}}{n \cdot n !}\left(\frac{\alpha^{\star}}{\alpha_{0}}\right)^{n}-\mathrm{I}\right] .
\end{aligned}
$$

Equations (22) and (23) give the pressure jump across the wave. Equation (19) can be used to calculate the wave profile, i.e. the variation of the density ratio $\alpha$ inside the stress wave. Inversion of this equation results with

$$
\eta=\int_{\alpha_{0}}^{\alpha} \frac{\mathrm{d} \beta}{\mathrm{F}\left(\beta, \alpha_{0}, C_{0}\right)}
$$

where

$$
\begin{aligned}
\mathbf{F}\left(\beta, \alpha_{0}, C_{0}\right)=\frac{\mathrm{I}}{\gamma C_{0}}\left\{\frac{4 \mathcal{J} Y_{0}}{3 \mathrm{f}(\beta)} \int_{\alpha_{0}}^{\beta} \exp \left(-\phi \beta^{\prime} / \alpha_{0}\right) \ln \left(\frac{\beta^{\prime}}{\beta^{\prime}-\mathrm{I}}\right) \mathrm{d} \beta^{\prime}+\right. \\
\left.\quad+\frac{2 \rho_{0} C_{0}{ }^{2}}{3 \alpha_{0} \mathrm{f}(\beta)}\left(\beta-\alpha_{0}\right)\left[\beta^{2}-\frac{\alpha_{0}}{2}\left(\beta+\alpha_{0}\right)\right]\right\}^{\frac{1}{2}} .
\end{aligned}
$$

Setting $\alpha=\alpha^{\star}$ in Equation (24) then gives $\eta_{1}$, the length of the wave, but this equation can also be used to calculate the $\alpha-\eta$ profile during the passing of the wave. Equation (14) can then be used to calculate the pressure profile and Equation (I3) then gives the velocity profile for the particle. 


\section{COMPARISON WITH EXPERIMENTAL RESULTS}

Napadensky (1964) conducted an extensive testing program on shock waves in snow at Camp Century. These experiments involved snow with densities generally higher than $500 \mathrm{~kg} \mathrm{~m}^{-3}$. The stress waves were generated by using a low-density explosive to drive a metal plate into solid cylindrical specimens. By varying the amount of explosive and the mass of the driver plate, a wide range of load conditions were achieved. The explosive technique however usually has a considerable amount of experimental scatter.

A streak camera was used to record the motion of etch marks on the snow specimen and the driver plate. In this way, both particle velocity $v$ and plastic wave velocity $C_{0}$ were recorded photographically. Since the speed of the camera was such that the exposure time was only about $5 \mu \mathrm{s}$, a fairly good time resolution was achieved. However, as indicated by Mellor (1977), large errors were incurred in the experimental program. There was very poor displacement resolution at the lower pressure levels, and it is questionable whether or not Napadensky's reduced data accurately reflect the actual stress-wave parameters. However, for the intermediate range of stress-wave pressures, the data appeared reasonable, since the streak film recordings both appeared to have good time resolution and were able to record particle displacement fairly accurately.

The material coefficients used in the constitutive law, as determined by Brown (in press), are

$$
Y_{0}=300 \text { bar }, \quad \mathcal{F}=3.07, \quad \phi=5.28 .
$$

Figures I and 2 give a direct comparison of the theory and Napadensky's experimental results, and, as can be seen the two compare fairly well. Figure I shows the pressure jump across the wave as a function of the density jump caused by the wave. Figure 2 shows the variation of wave velocity $C_{0}$ with particle velocity $v^{\star}$. For particle velocities above $20 \mathrm{~m} / \mathrm{s}$,

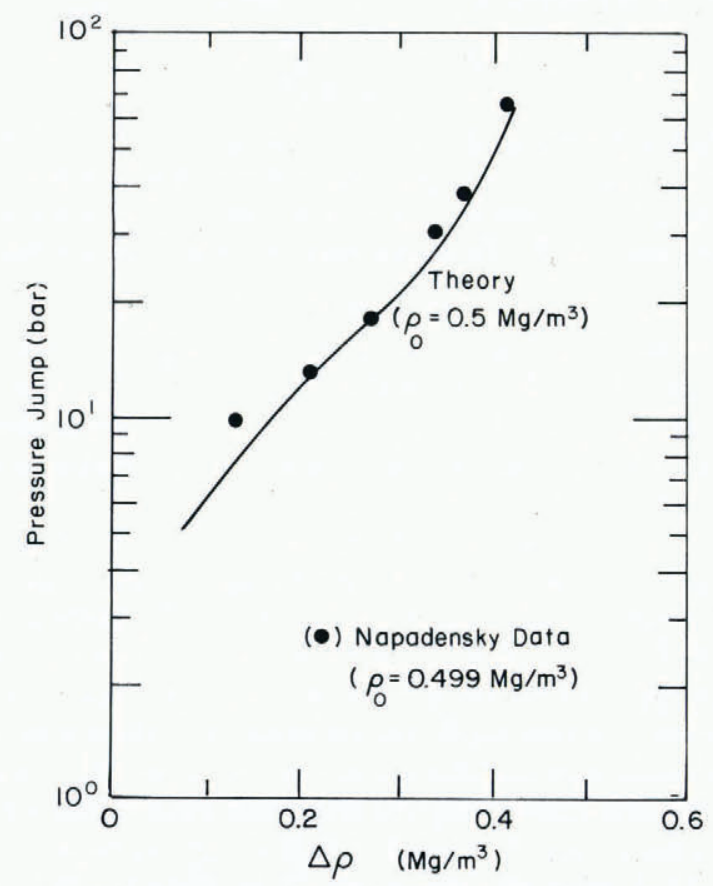

Fig. I. Pressure jump across wave as'a function of density jump. 


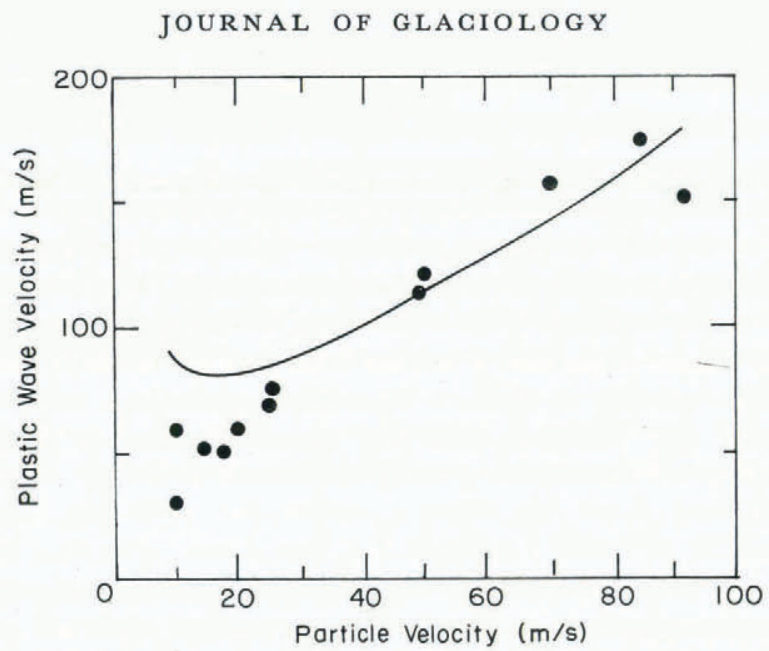

Fig. 2. Variation of plastic wave velocity with particle velocity.

the data and theory compare nicely, but below that, there is a significant deviation. The theoretical curve terminates at the point where the wave ceases to be fully plastic.

The data in Figure 2 at the lower particle velocities are questionable. Napadensky shows the plastic wave velocity decreasing to zero as the wave intensity (as indicated by $v^{\star}$, the particle velocity) decreases, and this result defies physical reasoning. As the wave intensity decreases, the severity of the plastic deformation also decreases. Accordingly the amount of the plastic deformation should become less significant, so that the pressure wave should begin to acquire characteristics of an elastic wave. From Equation (I4),

$$
C_{0}^{2}=\frac{\alpha_{0}}{\rho_{0}} \frac{\Delta \hat{p}^{\star}}{\Delta \alpha^{\star}} \text {. }
$$

If $\Delta \hat{p}^{\star} / \Delta \alpha^{\star}$ does not approach zero as the stress wave intensity is decreased, $C_{0}{ }^{2}$ must remain finite at low plastic strains. One would expect $\Delta \hat{p}^{\star} / \Delta \alpha^{\star}$ to acquire a value close to the elastic modulus. This argument is also supported by other analytical work on the theory of wave propagation in non-linear materials. In particular, we note the familiar equation

$$
C_{0}^{2}=E_{\mathrm{p}} / \rho_{0},
$$

where, for shock waves $E_{\mathrm{p}}$ is the instantaneous secant modulus, and for acceleration waves $E_{\mathrm{p}}$ is the instantaneous tangent modulus. In either stress wave, $E_{\mathrm{p}}$ increases as the degree of plastic deformation decreases, i.e. as a smaller percentage of the material is deformed plastically. As a result, $E_{\mathrm{p}}$ approaches $E$, Young's modulus of the material, and $C_{0}$ thereby increases to a value close to the elastic wave for weak plastic waves.

In the case of strong stress waves which produce complete plastic deformation of the matrix material and large jumps in density, the work-hardening characteristics of the material become important in determining the resulting wave speed. In particular $E_{\mathrm{p}}$ will increase as a result of densification and work-hardening effects, thereby increasing $C_{0}$ as indicated by Equation (27). Consequently, a very strong plastic wave will have a large wave speed. The above reasoning justifies the minimum calculated value of $C_{0}$ shown by the curve in Figure 2. This minimum is located near the transition zone between partial and fully plastic waves.

Figures 3 through 5 present results of parametric studies of stress waves in snow. Figure 3 shows how the density ratio varies through the front of the stress wave. For the less intense wave, a shorter wavelength is required to generate the acceleration forces necessary to produce the compaction to decrease $\alpha$ from 3.0 to 2.0 . 
Figure 4 illustrates the variation of pressure jump $\hat{p}^{\star}$ with density jump for a range of initial densities. Note that as the initial density is increased the curves are translated upward. Figure 5 also gives a very dramatic demonstration of the effects of work-hardening characteristics of snow. At low pressures, the plastic wave speeds are as expected, i.e. wave speed increases with density. But at higher pressures, this relationship is inverted, since large changes in $\alpha$ must accompany the large pressure jumps for snow with initial low densities. Consequently, there is considerable work-hardening, which has a stiffening effect, and this results in increased wave speeds. Snow with high initial densities would not undergo as much work-hardening.

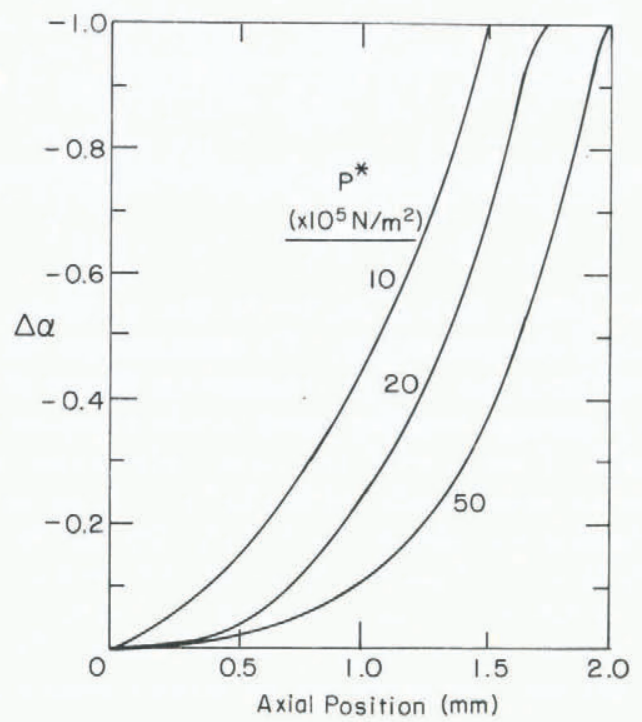

Fig. 3. Density-ratio profiles for plastic stress waves, $\rho_{0}=0.300 \mathrm{Mg} / \mathrm{m}^{3}, \Delta \alpha=-$ I.o.

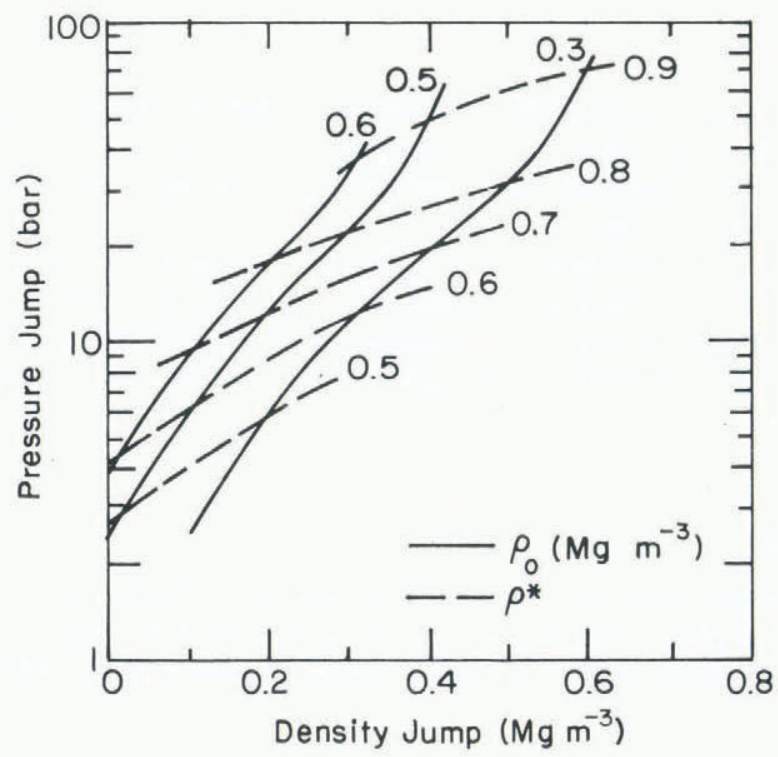

Fig. 4. Pressure jump across stress wave as a function of density jump. 


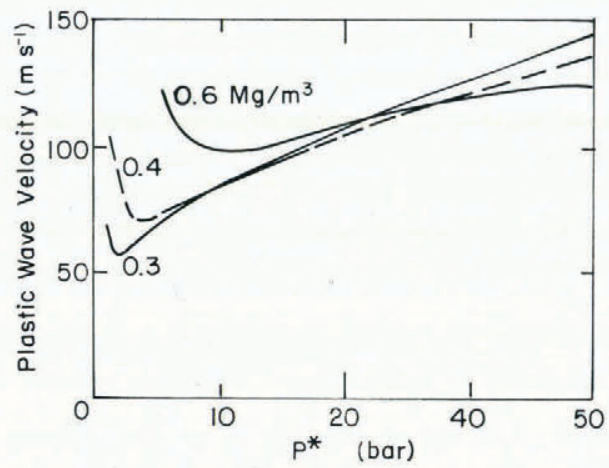

Fig. 5. Variation of plastic wave velocity with pressure jump across wave.

The results of this study give a partial answer to the questions concerning the large capacity of snow to absorb and dissipate energy. To demonstrate the ability of low-density snow to absorb energy, calculations were made to determine values of dissipated energy and kinetic energy produced by a pressure loading which would compact snow to bubbly ice at $900 \mathrm{~kg} \mathrm{~m}^{-3}$. For initial density of $300 \mathrm{~kg} \mathrm{~m}^{-3}$, the energy dissipation was found to be about $300 \%$ larger than for snow of an initial density of $600 \mathrm{~kg} \mathrm{~m}^{-3}$. In addition, the kinetic energy produced by the pressure loading was almost $700 \%$ more for the lower density snow, even after allowing for differences in mass. This points out the larger ability of the lower density snow to absorb energy during the process of compaction, a result due in part to the workhardening characteristics of the material. Other forms of energy dissipation not accounted for here would involve state changes occurring during impact, which would further enhance the energy-absorbing properties of snow.

\section{Conclusions}

The dynamic constitutive equation developed earlier by Brown (in press) has been shown adequately to represent the response of medium-to-high-density snow to plastic stress waves. Napadensky's data did show that the experimental results and theory were in good agreement except for weaker stress waves.

The constitutive law discussed here is considered to be valid for states of stress which are primarily hydrostatic. In situations where large deviatoric or shear stress-states are superimposed on the hydrostatic load, the material may behave differently. Fortunately, many cases exist where shearing stresses are significantly smaller than the pressures such as those studied in this paper.

\section{Acknowledgement}

The work reported here was supported by the U.S. Army Research Office, Grant No. DRXRO-RR P-I54I3GS. The author would like to express his appreciation to the Army Research Office and the U.S. Army Cold Regions Research and Engineering Laboratory for their support.

MS. received 2 January 1979 and in revised form 28 February 1979 


\section{REFERENCES}

Abele, G., and Gow, A. J. 1975. Compressibility characteristics of undisturbed snow. U.S. Cold Regions Research and Engineering Laboratory. Research Report 336.

Abele, G., and Gow, A. J. 1976. Compressibility characteristics of compacted snow. U.S. Cold Regions Research and Engineering Laboratory. Report 76-21.

Brown, R. L. In press. A volumetric constitutive law for snow subjected to large strains and strain rates. U.S. Cold Regions Research and Engineering Laboratory. Report.

Carroll, M. M., and Holt, A. C. 1972. Static and dynamic pore-collapse relations for ductile porous materials. Journal of Applied Physics, Vol. 43, No. 4, p. 1626-35.

Carroll, M. M., and Holt, A. C. 1973. Steady waves in ductile porous solids. Fournal of Applied Physics, Vol. 44, No. 10, p. $4386-92$.

Hawkes, I., and Mellor, M. 1972. Deformation and fracture of ice under uniaxial compression. Fournal of Glaciology, Vol. I I, No. 61, p. 103-31.

Mellor, M. [1975.] A review of basic snow mechanics. [Union Géodésique et Géophysique Internationale. Association Internationale des Sciences Hydrologiques. Commission des Neiges et Glaces.] Symposium. Mécanique de la neige. Actes du colloque de Grindelwald, avril 1974, p. 251-91. (IAHS-AISH Publication No. 114.)

Mellor, M. 1977. Engineering properties of snow. Fournal of Glaciology, Vol. 19, No. 81, p. I5-66.

Napadensky, H. 1964. Dynamic response of snow to high rates of loading. U.S. Cold Regions Research and Engineering Laboratory. Research Report 119. 